\title{
Contrôle de l'hémorragie, une compétence fondamentale : Un examen de la pression directe, des pansements, de l'insertion de pansement dans une blessure et des bandages pour sauver des vies
}

\author{
Par Christopher Picard, CD, BSN, RN, ENC(C)
}

L'hémorragie traumatique est « la principale cause des décès évitables » parmi les patients victimes d'un traumatisme (Spahn et al., 2013), causant 40 \% de toutes les mortalités (Curry et al., 2011). Quatre-vingtcinq pour cent de ces partients meurent avant de quitter les services d'urgence (Tien et al., 2007). Un contrôle amélioré de l'hémorragie permet d'améliorer de façon significative les résultats du patient (Kragh, 2009) et, par conséquent, le contrôle d'une exsanguination massive doit se produire avant le contrôle des voies respiratoires (Forest, Lax \& van der Velde, 2014). Les infirmiers/infirmières jouent un rôle très important dans l'identification et la gestion d'une hémorragie pouvant mettre une vie en danger; les compétences nécessaires pour un contrôle précoce de l'hémorragie sont des compétences infirmières. Ce document discutera des connaissances fondamentales associées aux interventions de base du contrôle de l'hémorragie. II explique également comment utiliser des manœuvres de base et comment accentuer les interventions de contrôle de l'hémorragie.

\section{Pression directe}

T a première étape dans le contrôle de l'hémorragie est l'application d'une pression directe. La pression directe est J'un des composants les moins recherchés du contrôle de l'hémorragie (Naimer et al., 2004). Les recommandations ne sont pas claires et son utilisation est souvent mal exécutée. Les principaux manuels sur les traumatismes recommandent la pression directe (Assid et al., 2014; Rotondo et al., 2012) mais ne vont pas plus loin. Ils n'expliquent pas la pression appropriée qu'il faut appliquer ni la durée, avec quoi il faut appuyer et comment remédier à des interventions inefficaces.

La recommandation la plus concrète sur la pression appropriée qu'il faut appliquer provient du Consensus Hartford du American College of Surgeons (ACS) : « Utilisez les deux mains...appuyez le plus fort possible » (Pons \& Jacobs, 2017). Cette déclaration de consensus est l'opinion d'un comité d'experts, mais n'est pas basée sur une preuve empirique et est difficile à normaliser entre les cliniciens. Avant cela, un objectif arbitraire de 60-90 mmHg pour le contrôle de l'hémorragie par pression directe avait été suggéré (Naimer et al., 2004). Même si aucune réelle justification n'a été fournie, cet article offre la seule pression quantifiable pour aider les patients victimes d'un traumatisme.

Les curriculums de soins avancés de réanimation traumatologique et les cours obligatoires en soins infirmiers de traumatologie ne mentionnent aucune durée minimale pour le maintien de la pression (Assid et al., 2014; Rotondo et al., 2012). Les recommandations empiriques sur la durée du maintien de la pression sont assez vagues. Les documents en soins infirmiers suggèrent un minimum de cinq à dix minutes (Day, 2016), et le consensus européen des médecins recommande de passer à des mesures plus agressives si le contrôle de l'hémorragie n'est pas réussi en une minute (Forrest, Lax \& van der Velde, 2014). Cependant, aucun n'offre de preuves ou de raison claire à ces recommandations. Une méta-analyse des dispositifs hémostatiques d'angiographie peut offrir une meilleure preuve de la durée minimale d'une pression, en concluant que les dispositifs hémostatiques ont un meilleur temps à l'hémostase avec une moyenne d'environ 20 minutes; mais la portée varie considérablement (Dahal et al., 2017). Même si ces données ne sont pas spécifiques au traumatisme, elles peuvent être utilisées comme guide pour les patients victimes d'un traumatisme. Cependant, il est important de noter que les patients d'angiographie sont moins susceptibles à avoir des coagulopathies induites par des traumatismes, et auront une ponction artérielle unique et plus petite à un endroit connu.

\section{Pansements}

Une pression directe est généralement appliquée en utilisant un pansement. Les pansements peuvent être fabriqués, ou improvisés, mais seront idéalement stériles, absorbants, non-adhésifs et non pelucheux. Même s'il existe différentes sortes de pansements pour contrôler une hémorragie, des pansements hémostatiques ou non-hémostatiques sont généralement utilisés. Les pansements hémostatiques contiennent des pro-coagulants pour faire accélérer le temps de coagulation. Ils fonctionnent de différentes façons, sont soutenus par des preuves raisonnables mais ne sont pas largement disponibles. Idéalement, l'utilisation de pansement hémostatiques augmentera mais ne sont pas 
encore largement utilisés. Par conséquent, cet article se concentrera sur l'utilisation de pansement non-hémostatiques.

Contrairement à plusieurs autres pansements spécialisés, la documentation comparant l'hémostase, les taux d'infection ou la facilité d'utilisation et les différents pansements traditionnels est insuffisantes. Il pourrait être possible d'assumer que tous les pansements traditionnels sont similaires. Les pansements de contrôle de l'hémorragie devraient permettre l'application et l'entretien de la pression directe sur la blessure, l'absorption et offrir une protection contre toute contamination. Les pansements ne doivent pas être appliqués en plusieurs couches (Forrest, Lax, van der Velde, 2014). L'application de pansements en plusieurs couches fait disperser la pression et peut faire accélérer les taux de saignement en augmentant l'action capillaire du pansement (Holley \& Filips, 2014). Les pansements sont une étape importante de l'application d'une pression directe, à condition qu'ils soient appliqués avec suffisamment de force. La saturation de pansements devrait déclencher une évaluation du contrôle de l'hémorragie, et non une application de plusieurs couches. Si le premier pansement devient saturé, d'autres mesures de contrôle de l'hémorragie doivent être mises en place pour s'assurer qu'une pression directe soit appliquée sur la blessure et pour vérifier si d'autres mesures doivent être utilisées, comme l'insertion d'un pansement dans la blessure ou l'application d'un garrot.

\section{Insertion de pansement dans une blessure}

Si la pression directe échoue, l'insertion d'un pansement dans une blessure serait la prochaine intervention à utiliser. Cette insertion de pansement augmente la pression directe sur les parties importantes de la blessure. L'ACS recommande l'insertion d'un pansement comme deuxième étape pour tout petit saignement non contrôlé à l'aide d'une pression directe, et comme première étape pour des blessures plus ouvertes ou proches du tronc et des membres (Pons \& Jacobs, 2017). Les preuves directes associées à l'insertion d'un pansement dans une blessure sont rares mais il y a d'excellentes preuves de cette méthode lors de chirurgies de sauvetage (Spahn et al., 2013). Cette méthode d'insertion du pansement pour des blessures associées à un traumatisme est assez similaire à celle utilisée sur des blessures chroniques, chirurgicales ou abcès, à l'exception de blessures bien serrées. Pour appliquer cette méthode, le tissu propre, la gaze ou le pansement hémostatique doit être inséré le plus profondément et fermement possible dans la blessure. Cette insertion doit être faite tout en maintenant une pression directe jusqu'à ce que la blessure soit entièrement remplie (Pons \& Jacob, 2017). Une fois le pansement inséré, la blessure doit être couverte avec un autre pansement, et une pression directe très importante doit être maintenue semestriellement ou par le biais d'un bandage serré.

\section{Bandages}

Il est parfois difficile au personnel dédié de maintenir une pression suffisamment forte pendant une durée appropriée. C'est pour cette raison que le bandage est souvent utilisé pour maintenir cette pression. Des données expérimentales suggèrent qu'un bandage élastique permet de maintenir une pression directe suffisamment importante sur le lit de la plaie plutôt que des pansements inélastiques (3 $3 \mathrm{mmHg}$ ) (Naimer et al, 2004). Des pansements spécialisés avec un bandage élasticisé intégré et une « barre de pression » pour exercer une pression sont disponibles. Des données expérimentales montrent que les barres intégrées peuvent entraîner une augmentation significative de la pression directe (Shipman \& Lessard, 2009). Des rapports de cas suggèrent que des « barres de pression » improvisées peuvent être bénéfiques (Rudge, Rudge \& Rudge, 2010), et ces pansements ont été largement utilisés par les militaires occidentaux avec de bons résultats. Cependant, il y a très peu de données expérimentales démontrant leur supériorité face aux autres sortes de pansements. Les recherches indiquent que les bandages élasticisés peuvent être utilisés en toute sécurité sans appliquer un garrot sur les membres. Lorsque l'on compare cela à une pression directe et un bandage en tissu, les bandages élasticisés maintiennent une pression plus élevée et constante, et restent mieux en place sans appliquer un garrot sur les membres (Naimer et al, 2004; Shipman \& Lessard, 2009). Reproduire ces bandages peut être facilement fait avec une réserve normale en appuyant fermement la gaze à la blessure, en appliquant un bandage élastique autour aussi fermement que possible au-dessus d'une toute petite zone avec une tension croissante. Les bandages doivent être vérifiés régulièrement pour s'assurer qu'il n'y a aucun saignement chez les patients à travers les pansements, que le bandage reste bien serré et pour contrôler la circulation en aval.

\section{Garrots}

Même si les garrots ont été utilisés avec succès durant des siècles, ce n'est que récemment qu'ils sont considérés comme une mesure de dernier recours en raison du risque perçu pour un membre endommagé. (Kragh 2009). D’excellentes preuves ont démontré l'efficacité du garrot dans le contrôle de l'hémorragie. Si une blessure ne répond pas à une pression directe ou à l'insertion d'un pansement, les garrots doivent être vus comme la prochaine étape. L'hémorragie d'extrémité est la principale source de décès évitable dans les forces armées. Leur expérience dans la gestion de ces types de blessures a démontré que les garrots étaient sûrs et efficaces, avec très peu de risques de complications (Kragh et al., 2009). Pour les hémorragies d'extrémité non-militaires, l'utilisation du garrot est également vue comme quelque chose de sûr et d'efficace (Scerbo et al., 2016).

Les garrots sont des bandes circonférentielles placées sur des os longs (jamais au-dessus d'une articulation) à proximité de blessures d'extrémité pour être serrées jusqu'à ce que le flux sanguin artériel (et donc le saignement) s'arrête. Les garrots peuvent être improvisés ou fabriqués commercialement. Cependant, les garrots commerciaux sont considérés comme étant de meilleure qualité que les dispositifs improvisés (Bulger et al., 2014). Improvisés ou mal utilisés, les garrots peuvent aggraver le saignement si la pression est insuffisante pour bloquer le flux artériel (Day, 2016). Les garrots uniquement veineux, comme un garrot pour commencer une IV ou prise de sang, entraîneront une augmentation de la pression veineuse, une dilatation et une accélération du saignement. Pour cette raison, utilisez un garrot commercial, si possible, serrez-le jusqu’à ce que le saignement 
s'arrête et les pulsations ne soient plus présentes, marquez le moment de son application et laissez le garrot en place jusquà ce que le contrôle du saignement soit atteint (Day, 2016). Si le contrôle de l'hémorragie n'est pas atteint avec un garrot, un garrot supplémentaire doit être placé à proximité et de la même façon.

\section{Conclusion}

Une hémorragie externe massive peut être mortelle en seulement quelques minutes et doit être gérée immédiatement. Les infirmiers/infirmières sont souvent les premiers en contact avec les patients entrant à l'hôpital et doivent souvent prendre des décisions rapides pour réussir à contrôler le saignement, et cela jusqu'à la mise en place d'une gestion définitive. Cet article a examiné les preuves d'une pression directe, de l'insertion d'un pansement dans une blessure, du bandage et de l'utilisation de garrots. Nous avons également discuté des connaissances fondamentales dans l'accélération des interventions de contrôle de l'hémorragie, et avons proposé des recommandations spécifiques sur la manière de mieux gérer l'hémorragie d'un traumatisme.

\section{Au sujet de l'auteur}

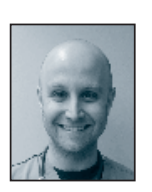

Christopher Picard, CD, BSN, RN, ENC(C), a travaillé dans des régions tertiaires, rurales et éloignées en tant qu'infirmier d'urgence. Il a également travaillé en tant que médecin pour les forces canadiennes dans des rôles pré-hospitaliers, cliniques et austères au Canada et à l'étranger. Il travaille présentement en tant qu'infirmier d'urgence à l'hôpital Royal Alexandra d'Edmonton $(A B)$. Ces sujets de recherche sont les soins en traumatologie, les pratiques axées sur les preuves et l'application des connaissances.

\section{RÉFÉRENCES}

Assid, P., Blank-Reid, C., Bokholdt, M., Brathcer, C., Cornell, A., ... Wolff, A. (2014). Trauma Nursing Core Course (TNCC) Provider Manual (7th Ed). Des Plains, IL. Emergency Nurses Association.

Bulger, E.M., Snyder, D., Schoelles, K., Gotschall, C., Dawson, D., Lang, E., ... White, L. (2014). An evidence-based prehospital guideline for external hemorrhage control: American College of Surgeons Committee on Trauma. Prehospital Emergency Care, 18(2), 163-173.

Curry, N., Hopewell, S., Dorée, C., Hyde, C., Brohi, K., \& Stanworth, S. (2011). The acute management of trauma hemorrhage: A systematic review of randomized controlled trials. Critical care, 15(2), R92.

Dahal, K., Rijal, J., Shahukhal, R., Sharma, S., Watti, H., Azrin, M., ... Lee, J. (2017). Comparison of manual compression and vascular hemostasis devices after coronary angiography or percutaneous coronary intervention through femoral artery access: A meta-analysis of randomized controlled trials. Cardiovascular Revascularization Medicine, 19 August 2017. In Press.

Day, M.W. (2016). Control of traumatic extremity hemorrhage. Critical care nurse, 36(1), 40-51.

Forrest, M., Lax, P., \& van der Velde, J. (2014). Anesthesia, Trauma, and Critical Care Course Manual 2014. Retrieved from https:// www.ataccgroup.com/wp-content/uploads/2017/04/ATACCManual-version-8-low-resolution-v2.pdf

Holley, J., \& Filips, D. (2014). 10 hemorrhage control myths. Journal of Emergency Medical Services, 39, 12.

Kragh Jr, J.F., Walters, T.J., Baer, D.G., Fox, C J., Wade, C.E., Salinas, J., \& Holcomb, J.B. (2009). Survival with emergency tourniquet use to stop bleeding in major limb trauma. Annals of surgery, 249(1), 1-7.
Naimer, S.A., Anat, N., Katif, G., \& Team, R. (2004). Evaluation of techniques for treating the bleeding wound. Injury, 35(10), 974-979.

Pons, P., \& Jacobs, L. (2017). SAVE A LIFE: What everyone should know to stop bleeding after an injury. American College of Surgeons.

Rotondo, M., Fildes, J., Brasel, K., Kortbeek, J., Al Turki, S., ... McIntyre, C. (2012). Advanced Trauma Life Support (ATLS) Student Course Manual (9th Ed). Chicago, IL. American College of Surgeons.

Rudge, W.B., Rudge, B.C., \& Rudge, C.J. (2010). A useful technique for the control of bleeding following peripheral vascular injury. Annals of the Royal College of Surgeons of England, 92(1), 77.

Scerbo, M.H., Mumm, J.P., Gates, K., Love, J.D., Wade, C.E., Holcomb, J.B., \& Cotton, B.A. (2016). Safety and appropriateness of tourniquets in 105 civilians. Prehospital emergency care, 20(6), $712-722$.

Shipman, N. \& Lessard, C.S. (2009). Pressure applied by the emergency/Israeli bandage. Military Medicine, 174(1), 86-92.

Spahn, D.R., Bouillon, B., Cerny, V., Coats, T.J., Duranteau, J., Fernández-Mondéjar, E., ... Neugebauer, E. (2013). Management of bleeding and coagulopathy following major trauma: an updated European guideline. Critical care, 17(2), R76.

Tien, H.C., Spencer, F., Tremblay, L.N., Rizoli, S.B., \& Brenneman, F.D. (2007). Preventable deaths from hemorrhage at a level I Canadian trauma center. Journal of Trauma and Acute Care Surgery, 62(1), $142-146$. 